\title{
Integrated preclinical and clinical development of mTOR inhibitors in pancreatic cancer
}

\section{Garrido-Laguna', AC Tan', M Uson', M Angenendt', WW Ma', MC Villaroel', M Zhao', NV Rajeshkumar', A Jimeno', R Donehower', C lacobuzio-Donahue', M Barrett', MA Rudek', B Rubio-Viqueira ${ }^{3,4,5}$, D Laheru' and $M$ Hidalgo ${ }^{*, 3,4,5}$}

'The Sidney Kimmel Comprehensive Cancer Centre at Johns Hopkins and the Sol Goldman Pancreatic Cancer Research Center, The Johns Hopkins University, Baltimore, MD, USA; ${ }^{2}$ Oncogenomics Laboratory, TGen Research Institute, Phoenix, AZ, USA; ${ }^{3}$ Centro Integral Oncológico Clara Campal, Madrid, Spain; ${ }^{4}$ Facultad de Medicina, Universidad-CEU San Pablo, Madrid, Spain; ${ }^{5}$ Centro Nacional de Investigaciones Oncológicas, Melchor Fernandez Almagro 3, Madrid 28029, Spain

BACKGROUND: The purpose of this work was to determine the efficacy of inhibiting mammalian target of rapamycin (mTOR) in pancreatic cancer preclinical models and translate preclinical observations to the clinic.

METHODS: Temsirolimus ( $20 \mathrm{mg} \mathrm{Kg}^{-1}$ daily) was administered to freshly generated pancreatic cancer xenografts. Tumour growth inhibition was determined after 28 days. Xenografts were characterised at baseline by gene expression and comparative genomic hybridisation. Patients with advanced, gemcitabine-resistant pancreatic cancer were treated with sirolimus (5 mg daily). The primary end point was 6-month survival rate $(6 \mathrm{mSR})$. Correlative studies included immunohistochemistry assessment of pathway expression in baseline tumours, drug pharmacokinetics (PKs), response assessment by FDG-PET and pharmacodynamic effects in peripheralblood mononuclear cells (PBMCs).

RESULTS: In all, 4 of 17 xenografts (23\%) responded to treatment. Sensitive tumours were characterised by gene copy number variations and overexpression of genes leading to activation of the PI3K/AKt/mTOR pathway. Activation of p70S6K correlated with drug activity in the preclinical studies. Sirolimus was well tolerated in the clinic, showed predictable PKs, exerted pathway inhibition in post-treatment PBMCs and resulted in a $6 \mathrm{mSR}$ of $26 \%$. No correlation, however, was found between activated p70S6K in tumour tissues and anti-tumour effects.

CONCLUSION: Sirolimus activity in pancreatic cancer was marginal and not predicted by the selected biomarker.

British Journal of Cancer (2010) 1 03, 649-655. doi:10.1038/sj.bjc.66058I9 www.bjcancer.com

Published online 27 July 2010

(c) 2010 Cancer Research UK

Keywords: pancreatic cancer; mTOR; p70S6K; temsirolimus; sirolimus

Pancreatic cancer remains one of the most deadly cancers, ranking among the top causes of cancer-related deaths (Jemal et al, 2009). One of the reasons underlying the poor prognosis of this disease is the lack of effective systemic treatments. Patients with advanced pancreatic cancer are managed with gemcitabine-based combination chemotherapy with limited success (Moore et al, 2007). Attempts to develop new drugs in pancreatic cancer have, in general, focused on combination studies with gemcitabine, with very few single-agent screening trials being performed. Indeed, patients with gemcitabine-resistant disease have traditionally been considered too sick to participate in clinical trials and have been managed with palliative care. More recently, however, several phase II studies have shown that selected patients with gemcitabine-resistant pancreatic cancer can be safely treated with secondline chemotherapy (Gebbia et al, 2007; Kulke et al, 2007; Xiong et al, 2008; Wolpin et al, 2009). This observation opens the opportunity to test new agents in refractory patients

*Correspondence: Dr M Hidalgo; E-mail: mhidalgo@cnio.es Revised 24 June 2010; accepted 29 June 20I0; published online 27 July 2010 in single-agent phase II studies. However, strategies to select potentially active drugs and candidate biomarkers for a more effective clinical development are needed.

The mammalian target of rapamycin (mTOR) pathway has emerged as an important candidate pathway for drug development (Rubio-Viqueira and Hidalgo, 2006; Meric-Bernstam and Gonzalez-Angulo, 2009). In recent years, several inhibitors of this pathway have been developed in cancer and some compounds have been approved for selected indications. The mTOR is downstream in the PI3K/Akt signalling pathway and is activated in response to growth factor receptor activation and nutrient stimulation. The mTOR regulates p70S6K and 4EBP1, having an important role in cell-cycle control and cell proliferation. Pancreatic cancer is characterised by several key genetic alterations such as activating mutations in the KRAS oncogene or inactivation in the CDKN2A/INK4A tumour suppressor gene that result in abnormal cell signalling and altered control of cell proliferation (Jones et al, 2008). Thus, pancreatic cancer is, in principle, an attractive tumour type to test mTOR inhibitors, and indeed, preclinical studies in established pancreatic cancer cell lines support this notion (Grewe et al, 1999; Agbunag and Bar-Sagi, 2004; Ito et al, 2006). 
We have developed a set of freshly generated pancreatic cancer xenografts as a preclinical platform for preclinical screening and biomarker discovery in pancreatic cancer (Rubio-Viqueira et al, 2006). These tumours, which have been extensively characterised, represent the heterogeneity of the disease and retain the most important genetic features of the originator tumour (RubioViqueira et al, 2006; Rubio-Viqueira and Hidalgo, 2009). In this study, we explored the activity of mTOR inhibition in pancreatic cancer with the goals to determine whether the observed activity warranted clinical development and to prioritise biomarkers that could be incorporated in clinical trials.

\section{MATERIALS AND METHODS}

\section{Xenograft studies}

Direct pancreatic cancer xenografts were generated as previously reported (Rubio-Viqueira et al, 2006). In this study we used 17 xenografts from the Hopkins PancXenoBank collection. Temsirolimus (Torisel, Wyeth Pharmaceuticals, Philadelphia, PA, USA) was administered intraperitoneally at $20 \mathrm{mg} \mathrm{Kg}^{-1}$ daily for 28 days as previously reported (Ito et al, 2006). Tumour size was evaluated two times per week by caliper measurements using the following formula: Tumour volume $=\left(\right.$ length $\times$ width $\left.^{2}\right) / 2$. Relative tumour growth inhibition/regression was calculated as $T / C=\left(T_{\mathrm{i}}-T_{0} / C_{\mathrm{i}}-C_{0}\right), T_{\mathrm{i}}$ and $C_{\mathrm{i}}$ represent tumour size of treatment and control group at the end of experiments, respectively; $T_{0}$ and $C_{0}$ represent tumour size at initiation of experiments, respectively. $T / C>0$ represent growth inhibition, $T / C<0$ represents tumour regression. The research protocol was approved by the Johns Hopkins University Animal Care and Use Committee, and animals were maintained in accordance to guidelines of the American Association of Laboratory Animal Care.

\section{Microarray gene expression and array CGH profiling}

Xenografts were profiled at baseline for gene expression using Affymetrix U133 Plus 2.0 gene arrays (Santa Clara, CA, USA) in duplicates as reported (Jimeno et al, 2008c). Similarly, array comparative genomic hybridization $(\mathrm{CGH})$ was carried out as described (Barrett et al, 2004). Briefly, genomic DNA from normal and pancreatic cancer xenografts was fragmented and labelled according to published protocols (Wu et al, 2005). Labelled DNAs were hybridised to human Agilent 44A CGH microarrays consisting of $\sim 40000$ oligonucleotide probes (Agilent Technologies, Palo Alto, CA, USA) and scanned on an Agilent DNA microarray scanner. Raw $\log 2$ ratio data were calculated using Agilent Feature Extraction 9.1 software.

\section{Gene set enrichment analysis}

Gene set analysis was performed using the Gene set enrichment analysis (GSEA) software V2.0.2 (http://www.broad.mit.edu/gsea) (Subramanian et al, 2005). Genes represented by more than one probe were collapsed using the Collapse Probes utility to the probe with the maximum value. Gene set permutations were performed 500 times for each analysis, and the pathway/gene set list is sorted by the Normalized Enrichment Score. We used the pathways defined by the Kyoto Encyclopedia of Genes and Genomes (KEGG) database to determine the rank-ordered pathway list for the xenografts. Human pathway annotations were downloaded from KEGG (August 2007 release), and 166 gene sets passed the gene set size filter criteria $(\min =10, \max =500)$.

\section{ELISA}

Levels of phosphorylated p70S6K were quantified at baseline using a solid-phase sandwich ELISA as per manufacture instructions (Immunoassay Kit, cat. no. KHO0581, Invitrogen, Camarillo, CA, USA).

\section{Clinical study}

Patients with advanced pancreatic adenocarcinoma refractory to gemcitabine were eligible for this trial. Patients were required to have unidimensionally measurable disease and tumour tissue for immunohistochemistry (IHC) assessment or willingness to undergo a safe tumour biopsy. Other eligibility criteria included an Eastern Cooperative Oncology Group performance score 0-1, adequate haematological, renal and liver functions, including an absolute neutrophil count $>1500$ cells per $\mathrm{mm}^{3}$, haemoglobin $>9 \mathrm{~g}$ per $100 \mathrm{ml}$, serum creatinine $\leqslant 2 \mathrm{mg}$ per $100 \mathrm{ml}$, bilirubin $\leqslant 2 \mathrm{mg}$ per $100 \mathrm{ml}$, ALT, AST, alkaline phosphatase $\leqslant 5$ times the upper limit of normal and triglycerides and total cholesterol $<2$ times the upper limit of normal.

Sirolimus was administered at a single oral flat dose of $5 \mathrm{mg}$ per day continuously in an outpatient setting. A treatment cycle was of 28 days. Patients with grade 3 or 4 adverse events related to the study drug temporarily discontinued the treatment. They were asked to resume treatment on resolution of the toxic event to grade 0 or 1 at a reduced dose of $4 \mathrm{mg}$ per day. Patients experiencing second grade 3 or 4 toxicity were to undergo a second dose reduction to $3 \mathrm{mg}$ per day. In addition, patients who experienced symptomatic grade 2 toxicities or biochemical toxicity persisting for longer than 1 week had their daily dose of rapamycin decrease by one dose level to $4 \mathrm{mg}$ per day. Patients requiring more than two dose reductions were taken off study. Patients had complete blood count and chemistry tests performed at baseline and every week for the first cycle and at every other week thereafter. Toxicity was graded according to the National Cancer Institute Common Toxicity Criteria for Adverse Events Version 3.0. Response to treatment was measured using FDG-PET-CT scan for every other cycle.

\section{Pharmacokinetic sampling, analytical assay and data analysis}

Pharmacokinetic studies were performed after single and multiple doses during cycle 1 on days 1 and 28 . Whole blood was collected in EDTA-containing tubes pre-treatment and at 1, 2, 4 and $6 \mathrm{~h}$ post-treatment on days 1 and 28. Trough samples were collected before drug administration on days $2,3,8,15$ and 22 of the first cycle and on day 1 of the second cycle. Samples were stored at $-70^{\circ} \mathrm{C}$ or below. Sirolimus concentrations in whole blood were determined over a range of $0.5-200 \mathrm{ng} \mathrm{ml}^{-1}$ by a validated highperformance liquid chromatography with mass spectrometry detection (LC/MS/MS) method. Individual pharmacokinetic (PK) parameters were estimated by standard noncompartmental analysis using WINNonlin (Scientific Consultant, Apex, NC, USA) version 5.0 (Pharsight, Mountain View, CA, USA) (Gibaldi, 1982).

\section{Inmunohistochemistry of baseline tumour samples}

Immunohistochemical labelling was performed using standard methods. Formalin-fixed $5 \mu \mathrm{m}$ slides from paraffin-embedded tissue were deparafinised and stained with anti-phospho-p70S6K (Thr389) mouse monoclonal antibody (Cell Signaling Technologies). Immunolabelling was detected as per kit instructions (Ventana IVIEW Detection Kits, cat. no. 760091, Ventana). The intensity of the staining was determined using $H$-scores, a composite measure of intensity $\times$ proportion of staining cells.

\section{Correlative studies in peripheral-blood mononuclear cells}

Levels of total and phosphorylated [pT389] p70S6K in peripheralblood mononuclear cells (PBMCs) at baseline and at $6 \mathrm{~h}$ after the first sirolimus dose were quantified using a solid-phase sandwich ELISA as per manual instructions (Invitrogen Immunoassay kit, 
cat. no. KHO0581). The ratio of phospho/total p70S6K at $6 \mathrm{~h}$ after the first dose of sirolimus was normalised to baseline level and expressed as a percentage.

\section{Statistical considerations}

The primary end point of the clinical trial was the proportion of patients surviving at 6 months after treatment commencement (6-month survival rate $(6 \mathrm{mSR})$ ). The expected $6 \mathrm{mSR}$ with standard of care in the second-line setting is $30 \%$, and on the basis of the preclinical study, a $25 \%$ positive outcome was expected (Rothenberg et al, 1996). The sample size was determined to detect an improvement in $6 \mathrm{mSR}$ from 30 to $50 \%$. With 31 patients enroled and a one-side $\alpha$ of 0.05 , the study has a $76 \%$ power to detect this difference. The secondary objectives included: (a) to evaluate the relationship between baseline phospho-p70S6K expression by IHC and clinical outcome; (b) to characterise the toxicity and PKs of sirolimus in this patient population and; (c) to determine the pharmacodynamic effects of the agent on p70S6K activation in PBMCs. To test whether activation of PI3K/Akt/mTOR pathway was correlated with survival in patients treated with sirolimus, a Fisher's exact test was performed to determine the relation between phospho-p70S6K levels at baseline and 6mSR. Differences between PK parameters during sampling periods were compared by a Wilcoxon matched-pairs signed-rank test. All PK parameters are reported as mean \pm standard deviation unless otherwise noted. Pearson's correlation coefficient or Mann-Whitney $U$-tests were used to assess correlations between exposure $\left(C_{\max }\right.$ or AUC) and exploratory PD end points (that is, PBMC phospho-p70S6K, survival and PET-CT response). These tests were performed using JMP Statistical Discovery software (version 4.0.4; SAS Institute, Cary, NC, USA) or SPSS version 16 (SPSS, Chicago, IL, USA).

\section{RESULTS}

Tumour growth inhibition with temsirolimus in direct pancreatic cancer xenografts correlated with pathway activation

We observed tumour regression in four xenografts treated with temsirolimus (Figure 1). Assessment of gene copy number variations in these tumours by CGH array showed gene copy number variations that could potentially lead to PI3K/Akt/mTOR pathway activation. Xenografts Panc219 and Panc198 had focal gains in NRAS and KRAS, respectively, whereas Panc266 and Panc287 had a homozygous deletion of FHIT or PTEN, respectively (Figure 2A). These genetic abnormalities were not, however, specific because some of the resistant tumours also had similar genetic alterations such a FHIT losses and $A K T$ amplification. Supplementary Table 1 summarises the genomic alterations observed in these tumours.

To gain further insight into gene pathways that might predict response to temsirolimus, we performed GSEA on the gene expression profiles of both sensitive and resistant cases. From the GSEA results, there were 8 and 18 pathways with $P<0.01$ and false discovery rate $<20 \%$ enriched in the sensitive and resistant cases, respectively (Table 1). Five cancer (chronic myeloid leukaemia, glioma, pancreatic, prostate and renal cell cancer) pathways were among the pathways enriched in the sensitive cases. We found 32 common core genes that were upregulated in more than two pathways enriched in the sensitive cases (Supplementary Table 2). Many of these common core genes (AKT3, BRAF, EGFR, FGFR1, IGF1R, PIK3CA, PIK3R3, PDGFA and PDGFB) were related to the $\mathrm{PI} 3 \mathrm{~K} / \mathrm{Akt} / \mathrm{mTOR}$ pathway.

To further explore whether those genetic changes result in pathway activation, we measured phospho-p70S6K in the 17 xenografts using an ELISA test as a downstream read out of pathway activation. As shown in Figure 2B, there was a significant correlation between tumour regression after treatment with temsirolimus and baseline activation of p70S6K.

From these studies, we concluded that mTOR inhibitors exerted distinct anti-tumour effects in pancreatic cancer xenografts that were characterised by heightened activation of the PI3K/Akt/ mTOR pathway. To validate this hypothesis, we conducted a phase II trial with the mTOR inhibitor sirolimus in patients with advanced pancreatic cancer, integrating measurement of phosphop70S6K activation as a predictor of response.

\section{General clinical results}

A total of 31 patients, whose pertinent characteristics are listed in Table 2, were enroled in this trial. All patients had previously progressed to a gemcitabine-containing regimen, including 22 patients who had received previous chemotherapy for metastatic disease.

Overall treatment was well tolerated. The principal toxicities are summarised in Figure 3. Most of the adverse events were grade 1 (67\%), $24 \%$ were grade $2,9 \%$ were grade 3 and there was no grade

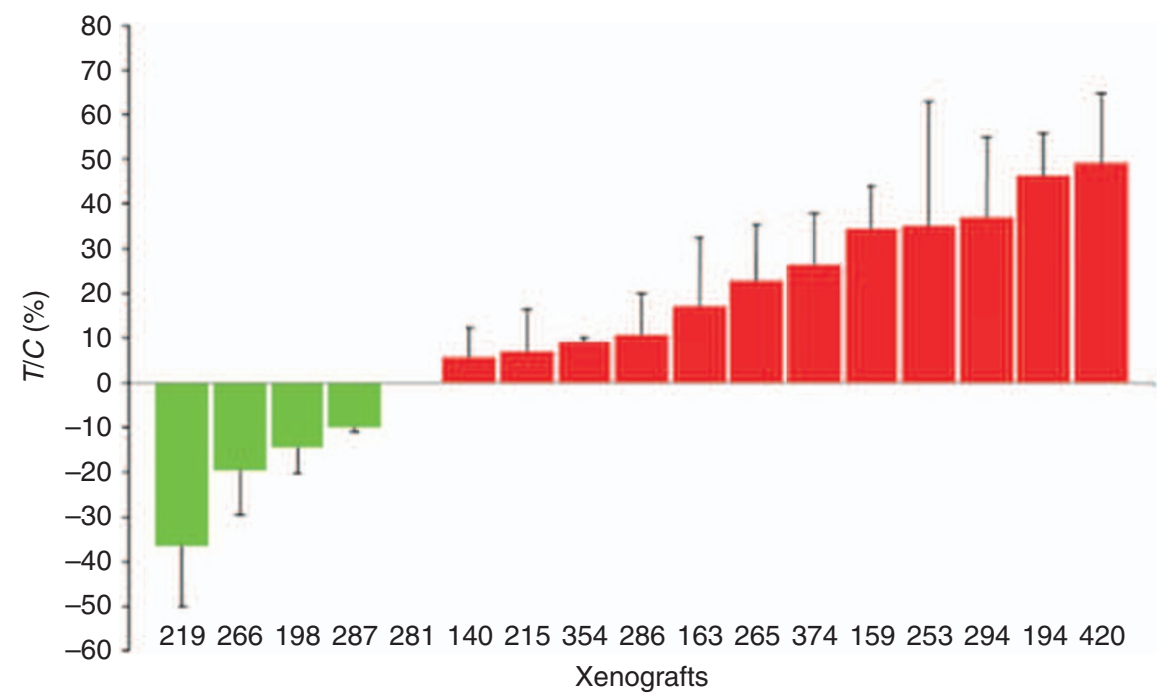

Figure I Tumour growth inhibition (T/C) in 17 direct pancreatic cancer xenografts treated with temsirolimus. Four xenografts showed tumour regression (negative T/Cs). Bars represent standard deviation. 

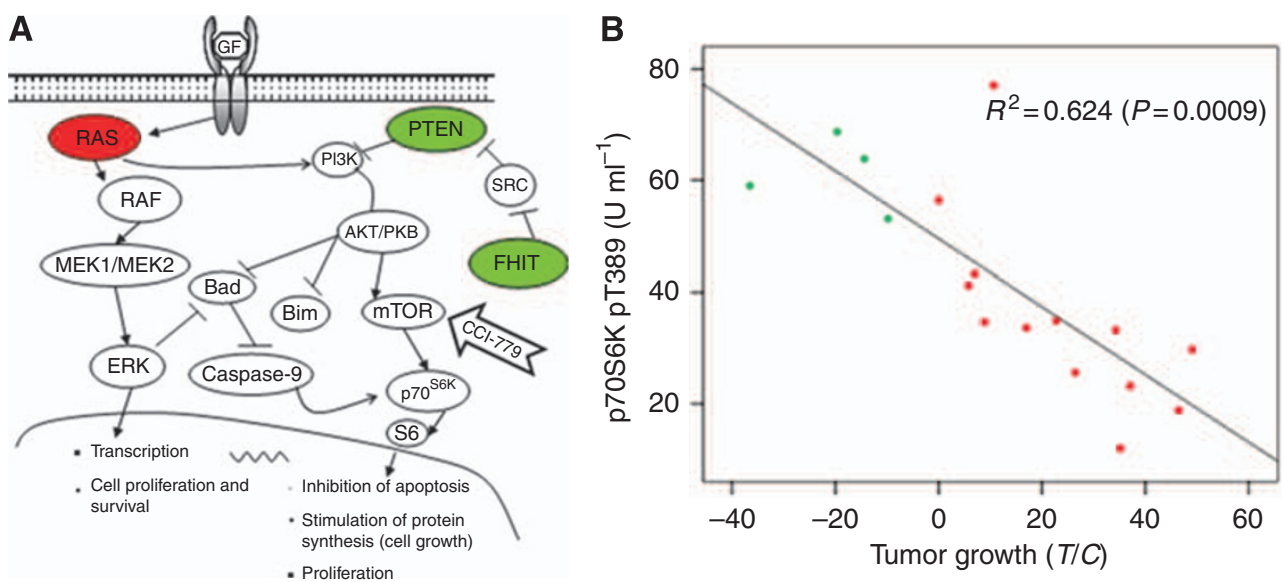

Figure 2 (A) Sensitive xenografts had focal gains in RAS, or homozygous deletions in PTEN or FHIT, potentially leading to pathway activation. Red colour represents gene copy number gains. Green colour represents gene copy number losses. (B) Tumour regression after treatment with temsirolimus in direct pancreatic cancer xenografts was correlated with baseline phospho-p7056K. Green dots represent xenografts that experienced tumour regression after temsirolimus treatment. Red dots represent xenografts that experienced tumour growth.

Table I Gene pathways enriched in temsirolimus-sensitive and -resistant xenografts as per KEGG classification

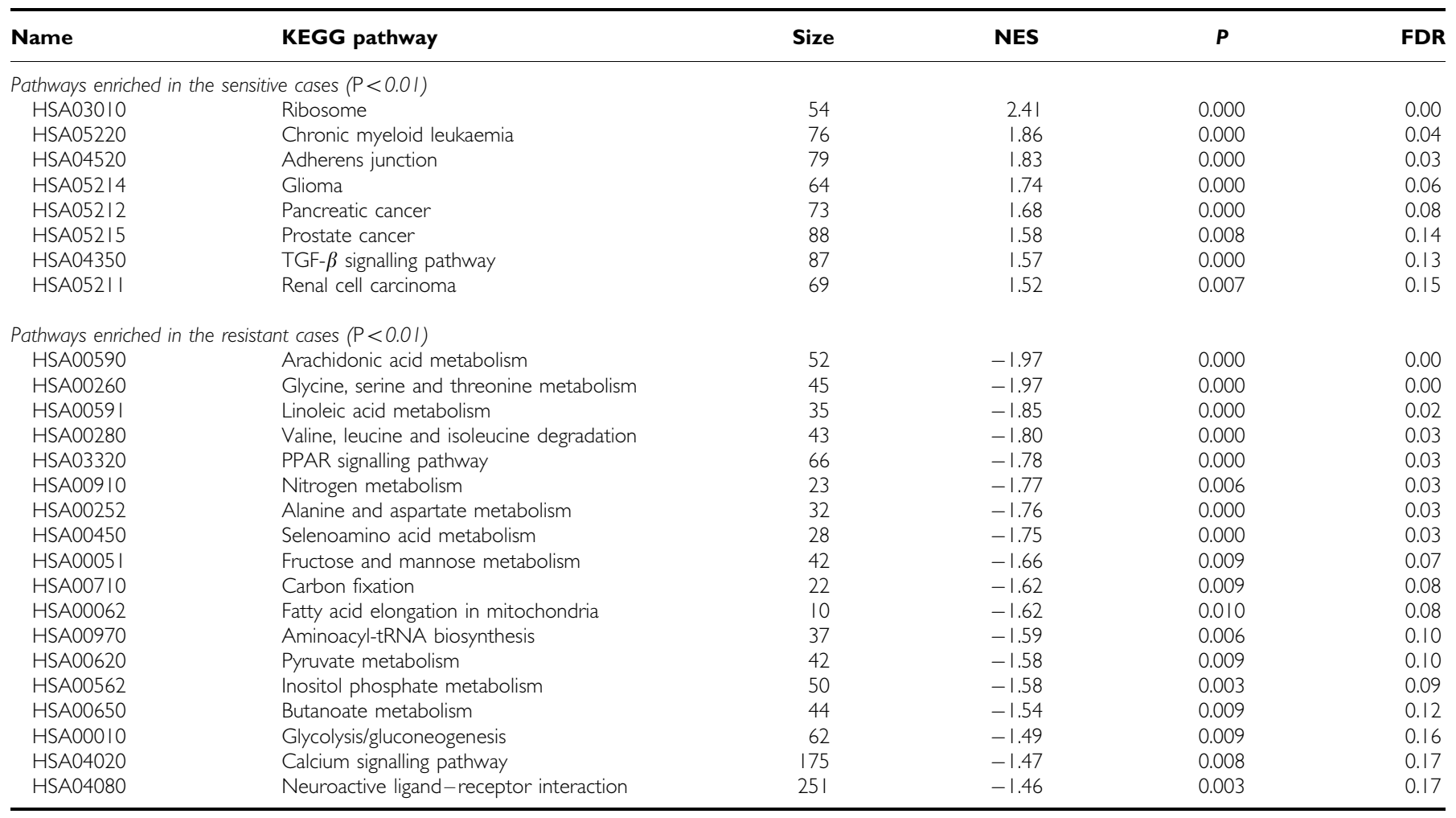

Abbreviations: $\mathrm{FDR}=$ false discovery rate; $\mathrm{KEGG}=$ Kyoto Encyclopedia of Genes and Genomes; NES= normalized enrichment score; $P=P$-value; TGF $=$ transforming growth factor.

4 adverse events. The most common grade 3 adverse event was hyperglycaemia, observed in $10 \%$ of the patients. Although 22 $(75 \%)$ patients had already received chemotherapy for metastatic disease before inclusion in this study, only one patient developed a grade 3 haematological adverse event (neutropenia). One patient developed a grade 3 increase in AST/ALT levels on cycle 1, day 15; treatment was stopped for 1 week with subsequent normalisation of liver function tests. One patient was admitted because of grade 3 enterocolitis on cycle 1, day 6; treatment was discontinued and the patient was discharged 4 days later. Other grade 3 toxicities were hyponatremia and rash in one patient $(3 \%)$ each.
There were no objective responses in this trial. Four patients (13\%) had stable disease (SD) at the 2-month follow-up evaluation. The $6 \mathrm{mSR}$ for the overall population was $26 \%$ (95\% CI $13-45 \%$; Supplementary Figure 1).

\section{PI3K/Akt/mTOR/pathway activation and clinical outcome}

We assessed baseline levels of phospho-p70S6K in 22 out of 31 patients. In contrast to the preclinical findings, there was no correlation between baseline levels of phospho-p70S6K by IHC and clinical outcome. 
Table 2 Patient's characteristics

\begin{tabular}{|c|c|c|}
\hline Characteristics & Number & Percentage \\
\hline \multicolumn{3}{|l|}{ Age (years) } \\
\hline Median & 64 & \\
\hline Range & $39-77$ & \\
\hline \multicolumn{3}{|l|}{ Sex } \\
\hline Male & 21 & 68 \\
\hline Female & 10 & 32 \\
\hline \multicolumn{3}{|l|}{ ECOG PS } \\
\hline 0 & 12 & 39 \\
\hline I & 19 & 61 \\
\hline \multicolumn{3}{|l|}{ Type of surgery } \\
\hline Whipple & 11 & 35 \\
\hline Pilorus preserving & 4 & 13 \\
\hline No previous surgery & 16 & 52 \\
\hline Previous perioperative chemoradiation & 11 & \\
\hline Previous perioperative chemotherapy & 4 & \\
\hline \multicolumn{3}{|l|}{ Previous chemotherapy for metastatic disease } \\
\hline GEM-Tarceva & 6 & 25 \\
\hline GEM & 5 & 21 \\
\hline GEM-XELODA & 3 & 12.5 \\
\hline GEM-CDDP & 3 & 12.5 \\
\hline GEM-LOHP & 3 & 12.5 \\
\hline GEM-CPTII & 1 & 4 \\
\hline GEM-XELODA-Txt & 1 & 4 \\
\hline GEM-Avastin & 1 & 4 \\
\hline XELODA-Avastin-Tarceva & । & 4 \\
\hline \multicolumn{3}{|l|}{ Serum Ca 19-9 $\left(\mathrm{Uml}^{-1}\right)$} \\
\hline Median & \multirow{2}{*}{\multicolumn{2}{|c|}{$\begin{array}{c}11048 \\
4-82808\end{array}$}} \\
\hline Range & & \\
\hline
\end{tabular}

Abbreviations: $\mathrm{CDDP}=$ cisplatin; $\mathrm{CPT}-\mathrm{I}$ | = irinotecan; $\mathrm{ECOG}$ PS = Eastern Cooperative Oncology Group performance score; GEM = gemcitabine; $L O H P=$ oxaliplatin; Txt= taxotere.

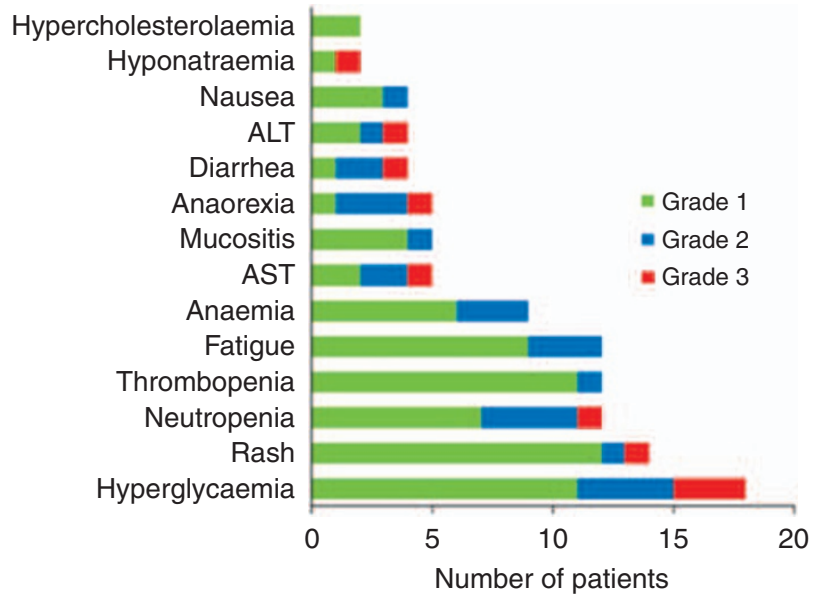

Figure 3 Treatment-related toxicities $(n=3 \mid)$.

\section{PK analysis}

PK data were evaluable in 30 patients. The PK profile of sirolimus was characterised by rapid absorption and a slow elimination phase after oral administration. By assessment of pre-treatment trough concentrations, steady state was reached by day 8 . Accumulation was noted as there was an increase in sirolimus exposure $\left(C_{\max } 15.5 \pm 9.2 \mathrm{ng} \mathrm{ml}^{-1}(n=30)\right.$ vs $24.8 \pm 10.4 \mathrm{ng} \mathrm{ml}^{-1}$ $(n=20)$ from day 1 to day 28 ; and day $1 \mathrm{AUC}_{\text {inf }} 234.5 \pm$ $121.5 \mathrm{ng} \mathrm{h} \mathrm{ml}^{-1}(n=22)$ vs day $28 \mathrm{AUC}_{0-24 \mathrm{~h}} 388.6 \pm 129.1 \mathrm{ng} \mathrm{h} \mathrm{ml}^{-1}$

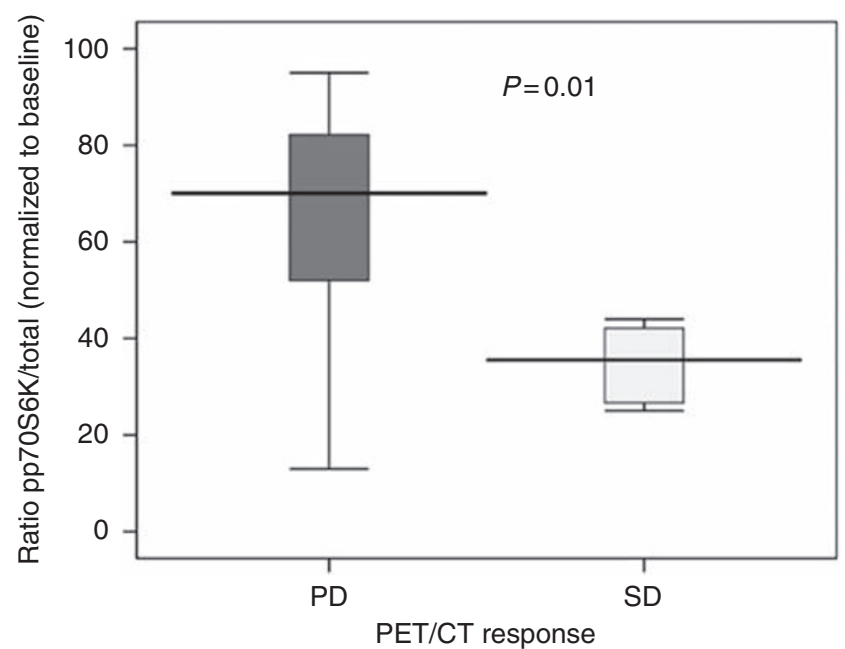

Figure 4 Activity of p70S6K at $6 \mathrm{~h}$ after first dose of sirolimus predicts stable disease on PET-CT evaluation. Patients with stable disease as shown by PET-CT on restaging at 8 weeks had greater inhibition of phosphop70S6K in PBMC at $6 \mathrm{~h}$ than those with disease progression $(P=0.0 \mathrm{l})$.

$(n=15) ;(P<0.05))$. There was a statistically significant increase in the day 28 half-life $(10.63 \pm 2.57 \mathrm{~h}(n=22) v s 20.67 \pm 5.12 \mathrm{~h}(n=14)$ on days 1 and 28, respectively; $P<0.05)$ and decrease in the apparent systemic clearance $\left(28.6 \pm 17.31 \mathrm{~h}^{-1} \quad(n=22) \quad v s\right.$ $13.6 \pm 4.0 \mathrm{lh}^{-1}(n=14)$ on days 1 and 28 , respectively; $\left.P<0.05\right)$. Sirolimus showed extensive distribution in excess of blood volume (Vz/F 414.6 \pm 206.81 (mean \pm s.d.; $n=22$ ) and $415.7 \pm 200.41$ $(n=14)$ on days 1 and 28 , respectively). The steady-state pretreatment trough concentration was $12.6 \pm 5.1 \mathrm{ng} \mathrm{ml}^{-1}(n=28)$. No correlation was found between $\mathrm{PK}$ parameters clinical outcome and PD markers.

\section{PD evaluation}

Patients with SD, as shown by PET-CT, had statistically significantly greater inhibition of p70S6K in PBMC at $6 \mathrm{~h}$ after treatment, as shown by a higher decrease in the ratio of phospho/ total p70S6K ( $35 v s 63 ; P=0.01$ ). Thus, activated phospho-p70S6K levels at $6 \mathrm{~h}$ were predictive for PET-CT response at 8 weeks (Figure 4).

\section{DISCUSSION}

This study aimed to determine the therapeutic role of inhibiting mTOR in preclinical models of pancreatic cancer and in patients with this disease. The mTOR inhibitor, temsirolimus, induced tumour regressions in 4 of 17 (23\%) freshly generated pancreatic cancer xenografts that were, in conjunction, characterised by genetic alterations, leading to an increased activation in the PI3K/Akt/mTOR pathway. As a single agent, sirolimus resulted in a $26 \% 6 \mathrm{mSR}$ in patients with previously treated pancreatic cancer, but did not result in tumour regressions in any patients. Contrary to our hypothesis, there was no indication that patients with higher activation of the pathway, as measured by the selected biomarker in this trial, did better.

The role of preclinical models in cancer drug development continues to evolve. In the era of cytotoxic agents, models were used to show tumour growth inhibition in a randomly selected group of rapidly growing xenograft models (Berger et al, 1990). More recent efforts, however, include testing agents in larger collection of xenografts representing a disease of interest, not only to gauge potential activity but also to understand predictors of efficacy (Perez-Soler et al, 2000; Rubio-Viqueira et al, 2006; 
Houghton et al, 2007; Jimeno et al, 2008a). With this goal in mind, we and others have started conducing large-scale phase II-like preclinical studies in pancreatic cancer. In this work, we followed such an approach to test the activity of mTOR inhibitors in pancreatic cancer.

One important consideration in this work, however, is that the level of activity in preclinical models that predict clinical efficacy is not established. Classically, a $T / C$ of $40 \%$ has been considered supportive of anti-tumour efficacy and used as a threshold to move drugs to the clinic. This criterion, in our opinion, is too unrestrictive and overestimates the expected clinical results. Indeed, if one applies the commonly used RECIST clinical criteria of response, a $T / C$ of $40 \%$ would be disease progression. We have therefore applied a more restrictive criterion and consider activity if there is a tumour regression. The current situation is that there are thousands of anti-cancer agents available but yet very little work in the clinic. A more selective preclinical approach is needed to prioritise which drugs to develop in patients. Efforts to better establish levels of preclinical efficacy that predict positive clinical outcome are, indeed, needed.

Another important, and not established, question is which level of preclinical activity is required to justify conducting a clinical trial. It is remarkable to note the important parallelisms between the preclinical and clinical study with $\sim 25 \%$ cases meeting the pre-specified primary objective in both studies. Notwithstanding that our patients were very heavily pretreated, a $25 \% 6 \mathrm{mSR}$ in the second-line pancreatic cancer is low and does not warrant further development of this drug as a single agent in this disease unless a predictive biomarker is identified. This has been indeed the finding of another recently published trial (Wolpin et al, 2009). In retrospect, we should have established a higher threshold of activity in the preclinical study before advancing the agent to clinical development. Given the larger number of available agents in clinical development and the limited resources, we propose that only agents with preclinical activity significantly greater than the clinical activity of interest are selected for clinical development.

The second major goal of this work was to identify biomarkers of activity. The preclinical data show that tumour regression after treatment with temsirolimus was limited to xenografts with high activation of the PI3K/Akt/mTOR pathway, as shown by high activation of p70S6K. There is a whole body of literature supporting the role of PTEN losses in activation of the PI3K/ $\mathrm{AKT} / \mathrm{mTOR}$ pathway in a variety of tumours including prostate breast and glioma (Li et al, 1997). Our finding that 1 of 17 (5\%) xenografts had PTEN deletion is in consonance with previous evidence that $P T E N$ losses are infrequent in pancreatic cancer (Okami et al, 1998). The fragile histidine triad (FHIT) gene, a tumour suppressor, is lost in most malignancies, including pancreatic cancer (Simon et al, 1998; Huebner and Croce, 2001). Loss of FHIT, leads to increase AKT activity both in vitro and in vivo (Semba et al, 2006). These findings are supported by the results from the GSEA, showing that sensitive xenografts were enriched in pathways with high content of genes involved in the $\mathrm{PI} 3 \mathrm{~K} / \mathrm{Akt} / \mathrm{mTOR}$ pathway. Thus, the striking correlation between the drug activity and pathway activation, as measured by phosphop70S6K, a downstream mediator of the pathway, is expected on the basis of the current knowledge of this pathway.

Although the overall level of activity of mTOR inhibitors in pancreatic cancer was modest, the finding that the activity could be linked to a biomarker was critical to support the conduction of the clinical study. If the $25 \%$ of patients who are sensitive can be identified upfront, the clinical development of the drug in pancreas cancer is feasible and likely to be successful. Unfortunately, we did not observe such a relationship in the clinical trial. Several factors can be considered to explain this issue. First, it could be that the concentrations of sirolimus achieved in patients are inferior to those achieved in mice. However, we have used the maximum tolerated dose of sirolimus based on data from a previous phase I study published by our group (Jimeno et al, 2008b). Second, PDA is characterised by an intense desmoplastic reaction that may lead to decreased intratumoural concentrations of sirolimus and thus explain lack of activity (Olive et al, 2009). Third, it could be that the biomarker selected, that is, activation of p70S6K, is not valid. We doubt, however, this is the reason. The notion that activation of the PI3K/Akt/mTOR pathway leads to p70S6K activation is supported by multiple studies (Hennessy et al, 2005; Riemenschneider et al, 2006). In addition, several trials with mTOR inhibitors suggest that this is a candidate marker (Galanis et al, 2005; Cloughesy et al, 2008). More likely, the negative results are because of trial design and technical reasons. We have used an IHC technique applied to archival tissues and we really do not know the preservation of the phosphorylated antigen over time in these materials. This is a recurrent problem in clinical trials that relay on archival tissue for which no satisfactory solution has been proposed. In addition to this, other investigators have questioned the performance of the specific antibody we used (anti-Thr389 S6K) for reliable quantification of the activity of p70S6K on paraffin sections (Cloughesy et al, 2008). We assessed p70S6K in primary tumours and treated metastatic disease. We do not know whether the process of generating tumour metastasis results in variations in this biomarker. In retrospect, a better strategy would have been to perform a fresh biopsy from a metastatic site and to use the same ELISA method that we have used in the preclinical study. It is clear that this approach adds morbidity, complexity and costs, but it may at the end be the only strategy to answer these questions. Finally, it could just mean that the preclinical model is not predictive at all of clinical activity and what is learnt in one does not predict what happens in the other.

In summary we have integrated a preclinical and clinical trial of mTOR inhibitors in pancreatic cancer. The agent resulted in $\sim 25 \%$ of cases achieving a positive response. In the preclinical study, activity was linked to pathway activation. This finding, however, was not observed in the clinic. The most likely explanation for this negative result is technical in nature, but other factors cannot be ruled out. In the absence of a well-defined biomarker to select patients, the overall level of activity does not suggest that these drugs as single agents would be effective in this disease.

\section{ACKNOWLEDGEMENTS}

This work has been supported by grant from FDA (FD-R-00252701) and Ignacio Garrido-Laguna 'Fundación Caja Madrid' fellowship.

Supplementary Information accompanies the paper on British Journal of Cancer website (http://www.nature.com/bjc)

\section{REFERENCES}

Agbunag C, Bar-Sagi D (2004) Oncogenic K-ras drives cell cycle progression and phenotypic conversion of primary pancreatic duct epithelial cells. Cancer Res 64: 5659-5663
Barrett MT, Scheffer A, Ben-Dor A, Sampas N, Lipson D, Kincaid R, Tsang P, Curry B, Baird K, Meltzer PS, Yakhini Z, Bruhn L, Laderman S (2004) Comparative genomic hybridization using oligonucleotide 
microarrays and total genomic DNA. Proc Natl Acad Sci USA 101: $17765-17770$

Berger DP, Fiebig HH, Winterhalter BR, Wallbrecher E, Henss H (1990) Preclinical phase II study of ifosfamide in human tumour xenografts in vivo. Cancer Chemother Pharmacol 26(Suppl): S7-S11

Cloughesy TF, Yoshimoto K, Nghiemphu P, Brown K, Dang J, Zhu S, Hsueh T, Chen Y, Wang W, Youngkin D, Liau L, Martin N, Becker D, Bergsneider M, Lai A, Green R, Oglesby T, Koleto M, Trent J, Horvath S, Mischel PS, Mellinghoff IK, Sawyers CL (2008) Antitumor activity of rapamycin in a Phase I trial for patients with recurrent PTEN-deficient glioblastoma. PLoS Med 5: e8

Galanis E, Buckner JC, Maurer MJ, Kreisberg JI, Ballman K, Boni J, Peralba JM, Jenkins RB, Dakhil SR, Morton RF, Jaeckle KA, Scheithauer BW, Dancey J, Hidalgo M, Walsh DJ (2005) Phase II trial of temsirolimus (CCI-779) in recurrent glioblastoma multiforme: a North Central Cancer Treatment Group Study. J Clin Oncol 23: 5294-5304

Gebbia V, Maiello E, Giuliani F, Borsellino N, Caruso M, Di Maggio G, Ferrau F, Bordonaro R, Verderame F, Tralongo P, Di Cristina L, Agueli R, Russo P, Colucci G (2007) Second-line chemotherapy in advanced pancreatic carcinoma: a multicenter survey of the Gruppo Oncologico Italia Meridionale on the activity and safety of the FOLFOX4 regimen in clinical practice. Ann Oncol 18(Suppl 6): vil24-vi127

Gibaldi MPD (1982) Pharmacokinetics, 2nd edn, Dekker: New York, NY

Grewe M, Gansauge F, Schmid RM, Adler G, Seufferlein T (1999) Regulation of cell growth and cyclin D1 expression by the constitutively active FRAP-p70s6K pathway in human pancreatic cancer cells. Cancer Res 59: $3581-3587$

Hennessy BT, Smith DL, Ram PT, Lu Y, Mills GB (2005) Exploiting the PI3K/AKT pathway for cancer drug discovery. Nat Rev 4: 988-1004

Houghton PJ, Morton CL, Tucker C, Payne D, Favours E, Cole C, Gorlick R, Kolb EA, Zhang W, Lock R, Carol H, Tajbakhsh M, Reynolds CP, Maris JM, Courtright J, Keir ST, Friedman HS, Stopford C, Zeidner J, Wu J, Liu T, Billups CA, Khan J, Ansher S, Zhang J, Smith MA (2007) The pediatric preclinical testing program: description of models and early testing results. Pediatr Blood Cancer 49: 928-940

Huebner K, Croce CM (2001) FRA3B and other common fragile sites: the weakest links. Nat Rev Cancer 1: 214-221

Ito D, Fujimoto K, Mori T, Kami K, Koizumi M, Toyoda E, Kawaguchi Y, Doi R (2006) In vivo antitumor effect of the mTOR inhibitor CCI-779 and gemcitabine in xenograft models of human pancreatic cancer. Int $J$ Cancer 118: $2337-2343$

Jemal A, Siegel R, Ward E, Hao Y, Xu J, Thun MJ (2009) Cancer statistics, 2009. CA Cancer J Clin 59: 225-249

Jimeno A, Li J, Messersmith WA, Laheru D, Rudek MA, Maniar M, Hidalgo M, Baker SD, Donehower RC (2008a) Phase I study of ON $01910 . \mathrm{Na}$, a novel modulator of the Polo-like kinase 1 pathway, in adult patients with solid tumors. J Clin Oncol 26: 5504-5510

Jimeno A, Rudek MA, Kulesza P, Ma WW, Wheelhouse J, Howard A, Khan Y, Zhao M, Jacene H, Messersmith WA, Laheru D, Donehower RC, Garrett-Mayer E, Baker SD, Hidalgo M (2008b) Pharmacodynamicguided modified continuous reassessment method-based, dose-finding study of rapamycin in adult patients with solid tumors. J Clin Oncol 26: $4172-4179$

Jimeno A, Tan AC, Coffa J, Rajeshkumar NV, Kulesza P, Rubio-Viqueira B, Wheelhouse J, Diosdado B, Messersmith WA, Iacobuzio-Donahue C, Maitra A, Varella-Garcia M, Hirsch FR, Meijer GA, Hidalgo M (2008c) Coordinated epidermal growth factor receptor pathway gene overexpression predicts epidermal growth factor receptor inhibitor sensitivity in pancreatic cancer. Cancer Res 68: $2841-2849$

Jones S, Zhang X, Parsons DW, Lin JC, Leary RJ, Angenendt P, Mankoo P, Carter H, Kamiyama H, Jimeno A, Hong SM, Fu B, Lin MT, Calhoun ES, Kamiyama M, Walter K, Nikolskaya T, Nikolsky Y, Hartigan J, Smith DR, Hidalgo M, Leach SD, Klein AP, Jaffee EM, Goggins M, Maitra A, Iacobuzio-Donahue C, Eshleman JR, Kern SE, Hruban RH, Karchin R, Papadopoulos N, Parmigiani G, Vogelstein B, Velculescu VE, Kinzler KW (2008) Core signaling pathways in human pancreatic cancers revealed by global genomic analyses. Science 321: $1801-1806$

Kulke MH, Blaszkowsky LS, Ryan DP, Clark JW, Meyerhardt JA, Zhu AX, Enzinger PC, Kwak EL, Muzikansky A, Lawrence C, Fuchs CS (2007) Capecitabine plus erlotinib in gemcitabine-refractory advanced pancreatic cancer. J Clin Oncol 25: 4787-4792

Li J, Yen C, Liaw D, Podsypanina K, Bose S, Wang SI, Puc J, Miliaresis C, Rodgers L, McCombie R, Bigner SH, Giovanella BC, Ittmann M, Tycko B,
Hibshoosh H, Wigler MH, Parsons R (1997) PTEN, a putative protein tyrosine phosphatase gene mutated in human brain, breast, and prostate cancer. Science 275: $1943-1947$

Meric-Bernstam F, Gonzalez-Angulo AM (2009) Targeting the mTOR signaling network for cancer therapy. J Clin Oncol 27: 2278-2287

Moore MJ, Goldstein D, Hamm J, Figer A, Hecht JR, Gallinger S, Au HJ, Murawa P, Walde D, Wolff RA, Campos D, Lim R, Ding K, Clark G, Voskoglou-Nomikos T, Ptasynski M, Parulekar W (2007) Erlotinib plus gemcitabine compared with gemcitabine alone in patients with advanced pancreatic cancer: a phase III trial of the National Cancer Institute of Canada Clinical Trials Group. J Clin Oncol 25: 1960-1966

Okami K, Wu L, Riggins G, Cairns P, Goggins M, Evron E, Halachmi N, Ahrendt SA, Reed AL, Hilgers W, Kern SE, Koch WM, Sidransky D, Jen J (1998) Analysis of PTEN/MMAC1 alterations in aerodigestive tract tumors. Cancer Res 58: 509-511

Olive KP, Jacobetz MA, Davidson CJ, Gopinathan A, McIntyre D, Honess D, Madhu B, Goldgraben MA, Caldwell ME, Allard D, Frese KK, Denicola G, Feig C, Combs C, Winter SP, Ireland-Zecchini H, Reichelt S, Howat WJ, Chang A, Dhara M, Wang L, Ruckert F, Grutzmann R, Pilarsky C, Izeradjene K, Hingorani SR, Huang P, Davies SE, Plunkett W, Egorin M, Hruban RH, Whitebread N, McGovern K, Adams J, Iacobuzio-Donahue C, Griffiths J, Tuveson DA (2009) Inhibition of Hedgehog signaling enhances delivery of chemotherapy in a mouse model of pancreatic cancer. Science 324: 1457-1461

Perez-Soler R, Kemp B, Wu QP, Mao L, Gomez J, ZeleniuchJacquotte A, Yee H, Lee JS, Jagirdar J, Ling YH (2000) Response and determinants of sensitivity to paclitaxel in human non-small cell lung cancer tumors heterotransplanted in nude mice. Clin Cancer Res 6: $4932-4938$

Riemenschneider MJ, Betensky RA, Pasedag SM, Louis DN (2006) AKT activation in human glioblastomas enhances proliferation via TSC2 and S6 kinase signaling. Cancer Res 66: 5618-5623

Rothenberg ML, Moore MJ, Cripps MC, Andersen JS, Portenoy RK, Burris III HA, Green MR, Tarassoff PG, Brown TD, Casper ES, Storniolo AM, Von Hoff DD (1996) A phase II trial of gemcitabine in patients with 5-FU-refractory pancreas cancer. Ann Oncol 7: 347-353

Rubio-Viqueira B, Hidalgo M (2006) Targeting mTOR for cancer treatment. Adv Exp Med Biol 587: 309-327

Rubio-Viqueira B, Hidalgo M (2009) Direct in vivo xenograft tumor model for predicting chemotherapeutic drug response in cancer patients. Clin Pharmacol Ther 85: 217-221

Rubio-Viqueira B, Jimeno A, Cusatis G, Zhang X, Iacobuzio-Donahue C, Karikari C, Shi C, Danenberg K, Danenberg PV, Kuramochi H, Tanaka K, Singh S, Salimi-Moosavi H, Bouraoud N, Amador ML, Altiok S, Kulesza P, Yeo C, Messersmith W, Eshleman J, Hruban RH, Maitra A, Hidalgo $M$ (2006) An in vivo platform for translational drug development in pancreatic cancer. Clin Cancer Res 12: $4652-4661$

Semba S, Trapasso F, Fabbri M, McCorkell KA, Volinia S, Druck T, Iliopoulos D, Pekarsky Y, Ishii H, Garrison PN, Barnes LD, Croce CM, Huebner K (2006) Fhit modulation of the Akt-survivin pathway in lung cancer cells: Fhit-tyrosine 114 (Y114) is essential. Oncogene 25: $2860-2872$

Simon B, Bartsch D, Barth P, Prasnikar N, Munch K, Blum A, Arnold R, Goke B (1998) Frequent abnormalities of the putative tumor suppressor gene FHIT at 3p14.2 in pancreatic carcinoma cell lines. Cancer Res 58: $1583-1587$

Subramanian A, Tamayo P, Mootha VK, Mukherjee S, Ebert BL, Gillette MA, Paulovich A, Pomeroy SL, Golub TR, Lander ES, Mesirov JP (2005) Gene set enrichment analysis: a knowledge-based approach for interpreting genome-wide expression profiles. Proc Natl Acad Sci USA 102: $15545-15550$

Wolpin BM, Hezel AF, Abrams T, Blaszkowsky LS, Meyerhardt JA, Chan JA, Enzinger PC, Allen B, Clark JW, Ryan DP, Fuchs CS (2009) Oral mTOR inhibitor everolimus in patients with gemcitabine-refractory metastatic pancreatic cancer. J Clin Oncol 27: 193-198

Wu G, Xing M, Mambo E, Huang X, Liu J, Guo Z, Chatterjee A, Goldenberg D, Gollin SM, Sukumar S, Trink B, Sidransky D (2005) Somatic mutation and gain of copy number of PIK3CA in human breast cancer. Breast Cancer Res 7: R609-R616

Xiong HQ, Varadhachary GR, Blais JC, Hess KR, Abbruzzese JL, Wolff RA (2008) Phase 2 trial of oxaliplatin plus capecitabine (XELOX) as secondline therapy for patients with advanced pancreatic cancer. Cancer 113: $2046-2052$ 